\title{
Type V collagen limits cardiac scar size
}

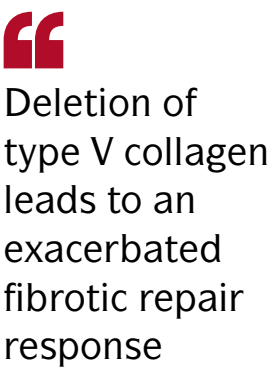

פ
Type V collagen, a minor constituent of the myocardial scar that forms after ischaemic injury, is an important regulator of scar size via a previously unrecognized role in limiting integrindependent fibrosis. "This is a very exciting new discovery showing that, in healing infarcts (and perhaps also in all healing wounds), induction of type $\mathrm{V}$ collagen is needed to deactivate fibroblasts," comments Nikolaos Frangogiannis (Albert Einstein College of Medicine, New York, NY, USA), who was not involved in the research.

Infarcted hearts in adult mammals have negligible regenerative capacity and heal by forming a scar. Nevertheless, this scar is not composed of dead cells but is living, dynamic tissue. Indeed, scar formation is a tightly regulated process, and scar size is a strong and independent predictor of outcomes in patients

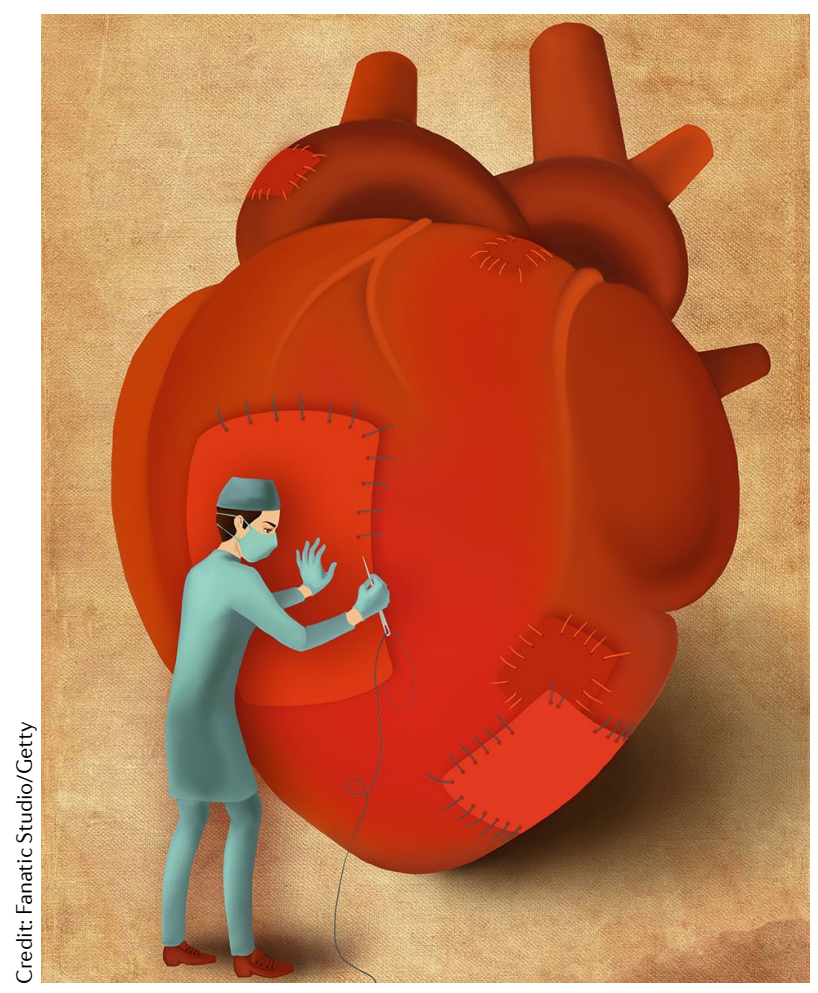

after myocardial infarction, with a large scar size and unrestrained fibrosis portending worse outcomes and the risk of heart failure. However, the mechanisms that regulate scar size are poorly understood, which motivated Arjun Deb and colleagues to study the factors that control the cardiac wound healing response.

The researchers induced ischaemic cardiac injury in mice and performed transcriptomic profiling of the resulting myocardial scars from 3 days to 6 weeks after injury. The scars were found to attain transcriptional maturity within the first 2 weeks. During this period, genes encoding collagens were among the most highly differentially upregulated.

In uninjured hearts, type I and type III collagens are abundantly expressed and comprise $90-95 \%$ of cardiac collagen. However, $>26$ different types of collagen have been described in mammals. The investigators found that type $\mathrm{V}$ collagen, which is minimally expressed in uninjured hearts, was upregulated after ischaemic injury in mice, although was still expressed only at relatively low levels. Importantly, knocking out one of the genes encoding type $\mathrm{V}$ collagen (Col5a1) resulted in a 1.5-fold increase in the fibrotic scar area and disrupted the normal architecture of the scar (shorter-length, irregular fibrillary structure with breaks in the collagen fibrils). "Deletion of type V collagen leads to an exacerbated fibrotic repair response," report the investigators.

The researchers next showed that type $\mathrm{V}$ collagen depletion altered the mechanical properties of the scar, leading to a reduction in scar stiffness. The cardiac fibroblasts from Col5a1 $1^{-/-}$ mice had increased expression of mechanosensitive $\alpha v \beta 3$ and $\alpha v \beta 5$ integrins, which drive myofibroblast differentiation, promote the production of extracellular matrix and increase scar size. Injection of cilengitide, a specific inhibitor of $\alpha v \beta 3$ and $\alpha v \beta 5$ integrins, significantly reduced scar size in $\mathrm{Col}_{5} \mathrm{1}^{-/-}$mice after ischaemic injury and rescued cardiac function.

These findings "lead to a model of wound healing whereby the structural constituents of scar tissue function to limit the size of the scar itself," concludes Deb. "The advantage of such a mechanism from an evolutionary point of view is to enable easy integration of the mechanical strength of the scar and the output of extracellular matrix."

The investigators observed genetic variation in Col5a1 expression in 100 strains of mice and hypothesize that differences in type $\mathrm{V}$ expression might exist after cardiac injury in humans. "We are testing whether we can develop type $\mathrm{V}$ collagen as a biomarker to predict wound healing outcomes," says Deb. Indeed, for patients who survive a large myocardial infarction, "application of type $\mathrm{V}$ collagen-containing patches may be an attractive therapeutic option," suggests Frangogiannis.

Individuals with classic EhlersDanlos syndrome have a mutation in one of the genes encoding type $\mathrm{V}$ collagen (typically either COL5A1 or COL5A2). These individuals have dysregulated wound healing, resulting in large scars. Deb and colleagues are currently testing whether targeting of specific integrins can attenuate scar size in animal models of Ehlers-Danlos syndrome. However, Frangogiannis advises caution about the potential therapeutic use of cilengitide after myocardial infarction because $\alpha v \beta 3$ and $\alpha v \beta 5$ integrins are ubiquitously expressed. Therefore, an inhibitor might have off-target adverse effects such as on angiogenesis, which is important for cardiac repair, so a precision medicine approach is needed.

Gregory B. Lim

ORIGINAL ARTICLE Yokota, T. et al. Type V collagen in scar tissue regulates the size of scar after heart injury. Cell https://doi.org/10.1016/ j.cell.2020.06.030 (2020) 